\section{Simulación clínica colaborativa para el desarrollo de competencias de trabajo en equipo en estudiantes de medicina}

\author{
SERGIO GUINEZ-MOLINOS ${ }^{1, \mathrm{a}}$, PATRICIO MARAGAÑO LIZAMA ${ }^{1}$, \\ CARMEN GOMAR-SANCHO
}

\section{Collaborative clinical simulation to train medical students}

In health sciences and medicine, collaborative learning has an important role in the development of competences to solve clinical situations. Adequate cooperation, coordination and communication skills have a direct effect on patient safety. Computer Supported Collaborative Learning (CSCL) and Clinical Simulation (CS), separately, are effective and efficient educational methods to develop competences in undergraduate medical students. To our knowledge, educational models that combine both teaching methods, including a personalized attention of the student, educational infrastructure, materials, teaching techniques and assessment competencies, have not been proposed previously. This article describes the application of a combined model of CSCL and CS for teaching clinical competences to medical students. Since 2015, the collaborative clinical simulation model is part of the training agenda of the Universidad de Talca Medical School in Chile. During 2016 and 2017 it was also applied on students of the Universidad de Barcelona Faculty of Medicine in Spain. According to the experience acquired, implementation of this method is feasible with commonly used resources, although its real efficacy remains to be evaluated.

(Rev Med Chile 2018; 146: 643-652)

Key words: Education, Medical; Educational Measurement; High Fidelity Simulation Training; Psychometrics.

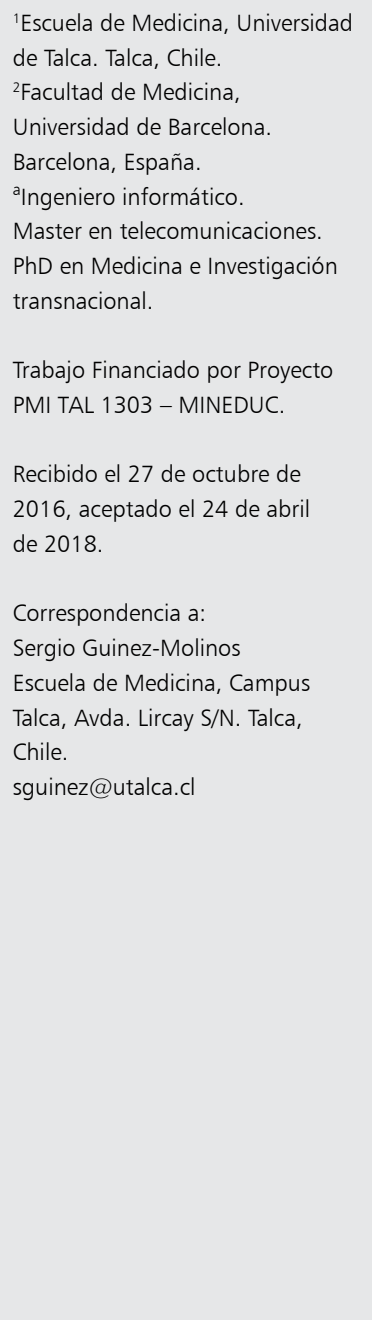

'Escuela de Medicina, Universidad

de Talca. Talca, Chile.

2Facultad de Medicina,

Master en telecomunicaciones.

$\mathrm{PhD}$ en Medicina e Investigación

Trabajo Financiado por Proyecto

Recibido el 27 de octubre de 2016, aceptado el 24 de abril

Sergio Guinez-Molinos

Escuela de Medicina, Campus

Chile.

guinez@utalca.c

\section{I} os profesionales de la salud realizan su trabajo mayoritariamente de modo colaborativo en equipos ${ }^{1}$. Esto los lleva a mantener un continuo aprendizaje en su vida profesional, desarrollando competencias de forma compartida, donde los miembros de un equipo aportan diferentes niveles de conocimientos y experiencias ${ }^{2}$. Las deficiencias del trabajo en equipo han sido reconocidas como la principal causa $(60-80 \%)$ en los errores y accidentes médicos ${ }^{3,4}$, que solo en Estados Unidos de Norteamérica se presenta como la tercera causa de muerte ${ }^{5}$.

La simulación clínica apoya el desarrollo de competencias clínicas de los estudiantes ${ }^{6-8}$. Sin em- bargo, se utiliza mayoritariamente para conseguir competencias de forma individual ${ }^{7,9}$, lo que no será la realidad del desarrollo profesional en un medio clínico. En este contexto, es importante enseñar las competencias médicas a los estudiantes dentro de ambientes de equipo y colaborativos ${ }^{2,9}$, incorporando formalmente este tipo de metodologías innovadoras al currículum ${ }^{10-12}$ y estimulando el trabajo en equipo.

En el presente trabajo, presentamos la aplicación de un innovador modelo de simulación clínica colaborativo ${ }^{13}$, combinando dos métodos aceptados de enseñanza: la simulación clínica y el aprendizaje colaborativo apoyado por tecnología. 
Este nuevo método busca solucionar algunas limitaciones de la simulación clínica tradicional, y su aplicación en las carreras de medicina de la Universidad de Talca y de la Universidad de Barcelona.

El principal objetivo del trabajo es demostrar, además de la aplicabilidad del modelo, la satisfacción percibida por los estudiantes. Esto fue planteado como fundamental y necesario para extender la aplicación en otros contextos y fortalecer la investigación en simulación clínica. La satisfacción es una unidad de medida importante para el aprendizaje comprometido y significativo, facilitando la participación activa y decidida en las experiencias de simulación ${ }^{14}$. También hay estudios en que la satisfacción de los estudiantes tiene correlación con el desempeño de los estudiantes ${ }^{15,16}$.

\section{Simulación clínica}

La simulación clínica se ha definido como: "la representación artificial de un proceso del mundo real, con la suficiente fidelidad para conseguir un objetivo específico y que, además, permite la valoración de su formación o de una determinada acción" . La simulación clínica permite la practica en entornos de aprendizaje simulados, permitiendo reducir las causas de eventos adversos ${ }^{17}$, como son los errores en la comunicación y trabajo en equipo ${ }^{4}$. Además, permite al alumno, corregir y tener presente la falta de experiencia clínica, reflexionar sobre su desempeño y aprender de sus errores, dentro de un ambiente controlado y seguro. Permite a profesores y alumnos aprender y desarrollar competencias de liderazgo, comunicación, coordinación de tareas y colaboración con otros profesionales de la salud en un entorno seguro $^{17}$.

La eficacia de la simulación clínica, al igual que múltiples actividades educacionales, proviene de la ejecución práctica de las acciones y autorreflexión del rendimiento para el aprendizaje y por ello se aplica a individuos o a grupos pequeños, lo que hace difícil hacerla extensiva a grupos numerosos como son los estudiantes de medicina ${ }^{18,19}$. Si la simulación clínica se desea integrar en los currículos hay cambiar su actual aplicación unidireccional, donde el académico es el centro de la actividad con un esfuerzo superlativo en la preparación, diseño, ejecución y debriefing de cada taller.
Aprendizaje colaborativo apoyado por tecnología

El CSCL (Computer Supported Collaborative Learning) orienta su investigación de las tecnologías de la información y comunicaciones (Tics) como herramientas de soporte, mediación, evaluación y monitorización de grupos de aprendizaje $^{20,21}$. El CSCL estudia la interacción dentro de un trabajo en grupo y cómo la colaboración y la tecnología facilitan el intercambio y distribución de conocimientos y experiencias entre los miembros de la comunidad de aprendizaje ${ }^{22}$. Los recursos tecnológicos en CSCL pueden presentarse como una simulación de alta fidelidad o cualquier entorno donde la tecnología es compatible con la colaboración ${ }^{23}$.

El aprendizaje entre pares es realmente una de las principales fuentes de aprendizaje de la vida. El aprendizaje entre pares es considerado de gran potencia formativa en la educación. En alumnos de medicina, la experiencia con aprendizaje entre pares ha tenido buenos resultado ${ }^{24,25}$. En el CSCL el aprendizaje entre pares es la base del aprendizaje colaborativo.

Principalmente, CSCL centra su atención en el aprendizaje a través de la colaboración entre estudiantes más que directamente del profesor ${ }^{26,27}$. Por esta razón, el rol de las Tics es brindar medios de comunicación y guía para lograr una interacción productiva entre los estudiantes. Esta forma básica de apoyo contempla simuladores y herramientas hardware/software especializados en esta área.

\section{Material y Método}

El modelo de simulación clínica colaborativo aplicado ${ }^{13}$ se diseñó considerando las fortalezas de los paradigmas de simulación clínica ${ }^{6,10}$ y aprendizaje colaborativo ${ }^{20,27,28}$, estructurado en 4 etapas secuenciales, con énfasis en el trabajo colaborativo de los participantes que componen la situación de aprendizaje .

De esta forma, se define la simulación clínica colaborativa como una metodología de enseñanza-aprendizaje para el desarrollo y evaluación de competencias clínicas, procedimentales, actitudinales y cognitivas; a través de pequeños grupos que trabajan colaborativamente en el diseño, ejecución y debriefing de casos simulados apoyados por la tecnología, insertos en una situación de aprendizaje colaborativo ${ }^{13}$. 


\section{Etapa 1. Diseño educacional (unidades académicas)}

El profesor, en coordinación y colaboración con su equipo académico, es el responsable de diseñar la práctica de simulación, estructurando cada una de las tres etapas siguientes. Este proceso de diseño comienza con la determinación de objetivos y estructura del taller ${ }^{29}$. Para esto, el profesor debe confeccionar el marco teórico y la guía clínica de simulación (material de estudio) que obligatoriamente debe tener, al menos, tres diagnósticos diferenciales (que serán la base del diseño colaborativo de cada escenario de simulación en la etapa 2 del proceso, entregándose un diagnóstico a desarrollar por cada grupo).

\section{Etapa 2. Diseño colaborativo}

En esta etapa presencial, se reúne el grupo de alumnos y el profesor encargado (PE) donde se procede a formar 3 subgrupos de trabajo (G1, G2, G3) que diseñarán cada uno de los casos clínicos a simular (Figura 1). Cada grupo se reúne (en salas diferentes) a diseñar un caso clínico, asignándoles 1 de los 3 diagnósticos diferenciales estudiados en la guía clínica de la Etapa 1. El profesor actúa de facilitador del proceso de diseño del caso, entregando consejos, aclarando dudas y guiando a cada uno de los grupos en la confección del caso de simulación clínica.

\section{Etapa 3. Simulación}

Una vez diseñados los escenarios, corresponde su ejecución. Cada grupo diseñador toma el rol de equipo instructor, apoyados por el profesor encargado (Figura 1). El grupo diseñador se asignan roles y responsables de las tareas dentro del escenario, posteriormente presenta el escenario de simulación clínica a sus compañeros (que será el grupo médico tratante), situándolos en un contexto específico, generando confianza y confort necesario para involucrarse en la simulación. Cuando finaliza cada uno de los escenarios de simulación de alta fidelidad, se observan las sensaciones y emociones del grupo médico tratante que acaba de finalizar su atención. Esto con el objetivo de tenerlos en consideración para la siguiente etapa.

\section{Etapa 4. Debriefing}

Debriefing es el término utilizado dentro de simulación clínica para referirse a una conversación entre dos o más personas que revisan un evento real o simulado, en la que los participantes analizan sus acciones y reflexionan sobre el papel de los procesos de pensamiento, habilidades psicomotricidades y estados emocionales para mejorar o mantener su rendimiento en el futuro $^{30}$.

En el debriefing colaborativo tienen participación los grupos diseñadores y los grupos médico tratante, moderados por el profesor, quien actúa como guía y estructura la reflexión del caso simulado. Basado en la estructura del modelo de debriefing sin juicio, creado por el Center for Medical Simulation de la Universidad de Harvard ${ }^{30}$, y el modelo de retroalimentación plus/delta propuesto por Motola ${ }^{11}$. Esta etapa refuerza el concepto de aprendizaje colaborativo. El profesor debe tomar un rol de guía del proceso, que modere la reflexión $y$ entregue antecedentes que permitan activar mecanismos de enseñanza entre los alumnos ${ }^{13}$.

En lo referente a los recursos humanos requeridos para el diseño y aplicación del modelo de simulación clínica colaborativa fueron los siguientes:

- 1 médico especialista/profesor de la asignatura correspondiente, encargado de la confección de las guías clínicas que permita el adecuado estudio de la competencia a simular.

- 1 instructor de simulación clínica, que brinde apoyo, evaluando los tiempos, y organizando la logística de su futura aplicación.

- 1 psicólogo cuantitativo experto en psicometría o evaluación de ítems, que permitiría la validación y confiabilidad de las dimensiones e ítems diseñados en los instrumentos.

Para el estudio exploratorio se realizaron 5 sesiones formativas (entre 9 y 15 participantes por sesión) de simulación clínica colaborativa de alta fidelidad a estudiantes de cuarto año de la Facultad de Medicina de la Universidad de Barcelona, en prácticas voluntarias del curso Malalties de l'Aparell Cardiocirculatori en el curso 2016-2017. En cada sesión participaba 1 profesor de la asignatura, 1 instructor de simulación y 1 metodólogo, quien guía la adecuada aplicación del modelo. Cada taller fue realizado en $3 \mathrm{~h}$ de duración, dividido en: 30 min para introducción del taller e inducción al simulador, $1 \mathrm{~h}$ para que cada grupo diseñara un escenario diferente: a) Taquicardia paroxística supraventricular; b) Flutter auricular y c) Taquicardia ventricular; $30 \mathrm{~min}$ 


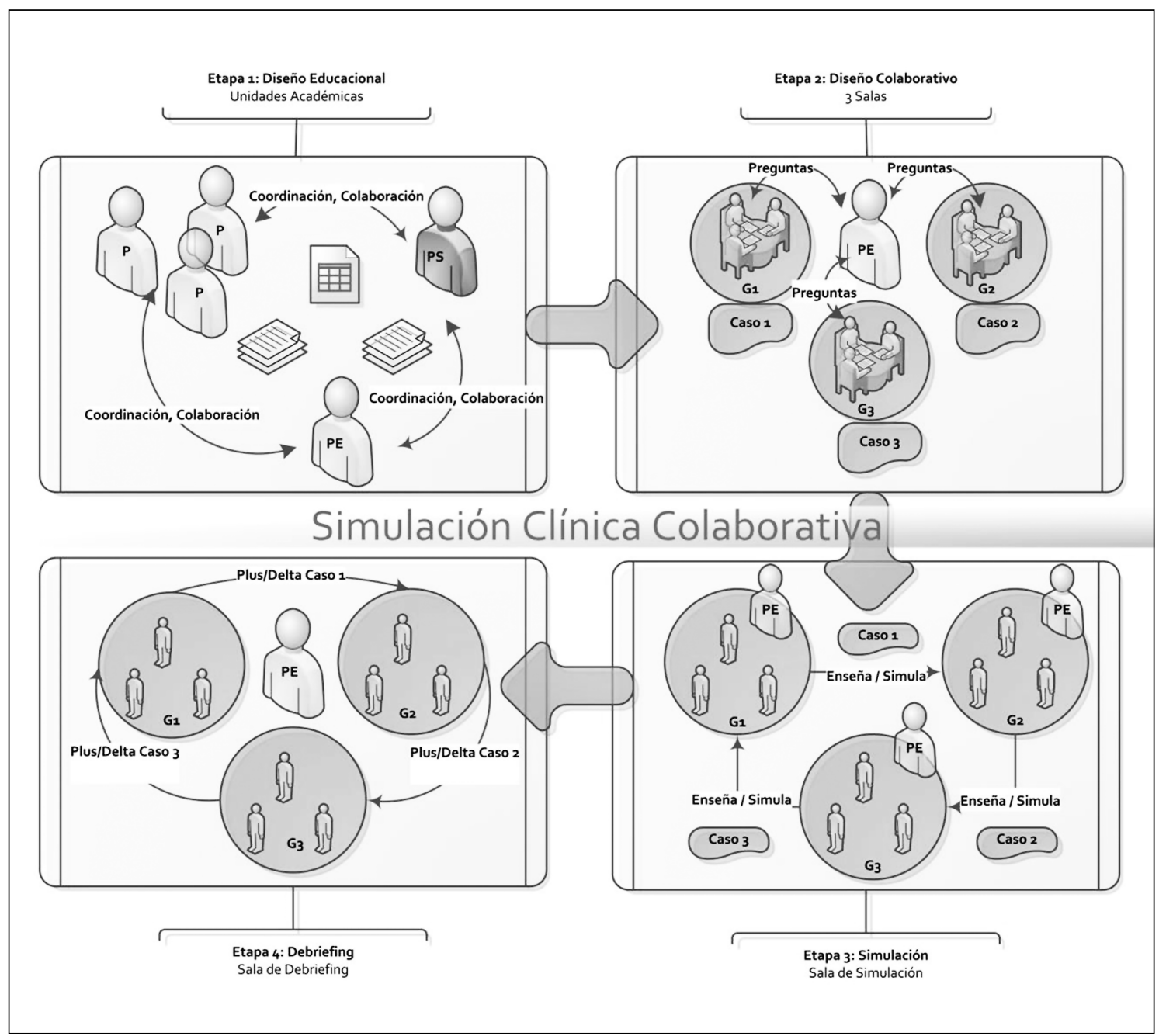

Figura 1. Modelo de Simulación Clínica Colaborativo. La etapa 1 es de coordinación y colaboración entre el profesor encargado $(P E)$, el equipo académico (P) y un experto en evaluación o psicometría (PS). Posteriormente, en la etapa presencial, cada uno de los tres grupos diseña y aplica sus escenarios para posteriormente participar en el debriefing colaborativo (etapas 2, 3 y 4).

para su simulación (10 min para cada grupo) y $1 \mathrm{~h}$ de debriefing.

El taller aplicado tenía como objetivo el diagnóstico y tratamiento de taquiarritmias en urgencias. En cada sesión se dividió el grupo en 3 subgrupos homogéneos (entre 3 y 5 participantes), donde cada grupo resolvió un escenario clínico simulado, diseñado, supervisado y evaluado por otro grupo. Los tres grupos realizaron el mismo proceso supervisados por un profesor instructor y un profesor de la asignatura. Cada sesión finalizaba con un debriefing común estructurado para incluir el diseño, la ejecución y evaluación de los casos, discutido colaborativamente entre los grupos.

Al finalizar cada taller en Barcelona, se le entregó a cada uno de los alumnos un cuestionario de satisfacción y, además, se les hizo una pregunta abierta: ¿Qué ventaja(s) formativa(s) de la simulación clínica colaborativa has percibido? Se analizaron todas las respuestas codificando y asociando a conceptos las frases, palabras y comentarios, con el objetivo de analizar ocurrencias y frecuencia de valoraciones. 


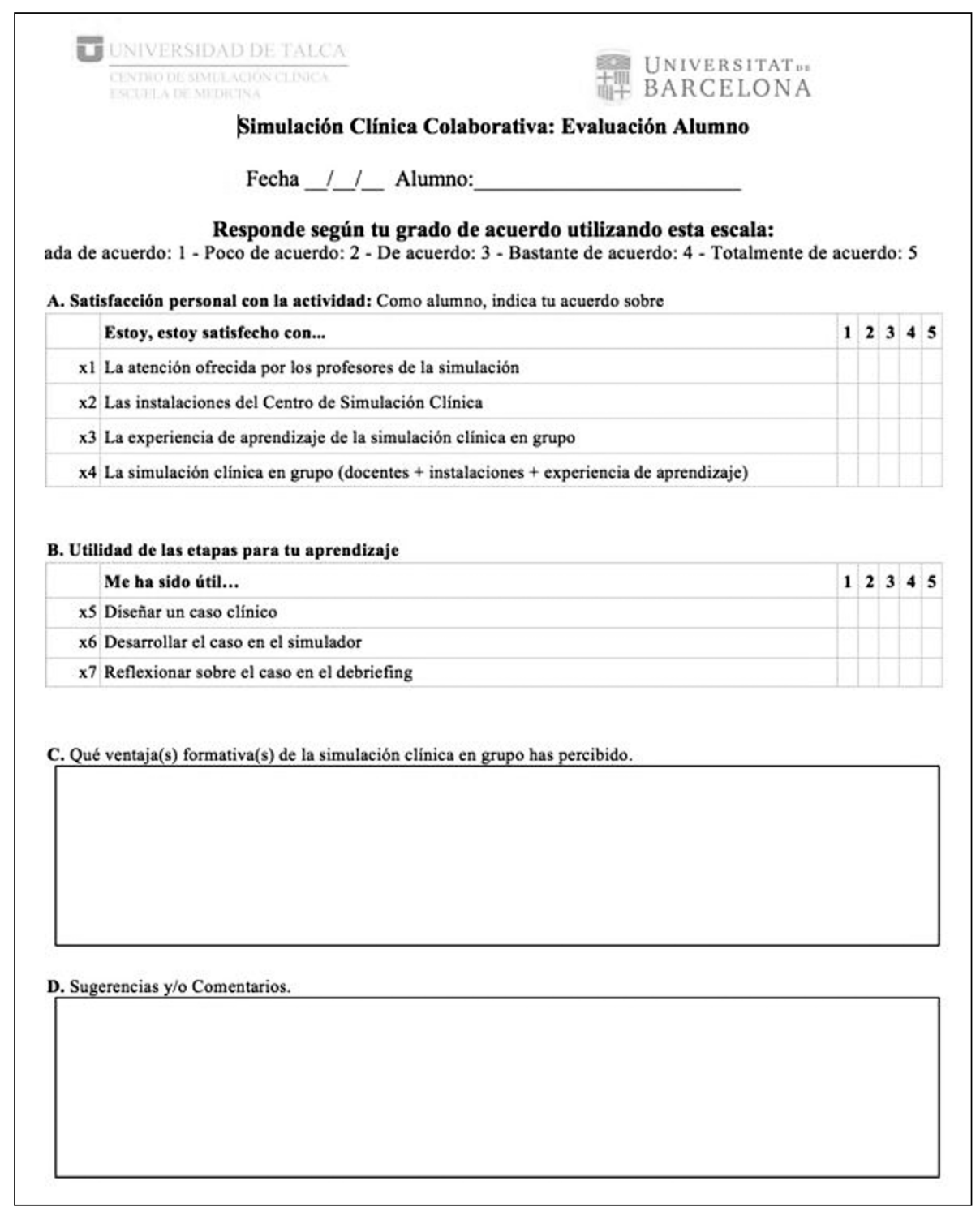

Figura 2. Cuestionario de satisfacción personal que cada estudiante contesta al finalizar el taller de simulación clínica colaborativo.

\section{$\underline{\text { Resultados de la aplicación piloto en Barcelona }}$}

El método es aplicable a dos contextos culturales distintos, el chileno y el español, en alumnos que cursaban el mismo año de su plan formativo. En la Universidad de Talca, el taller era obligatorio en la asignatura, pero en Barcelona la participación fue voluntaria con un alto grado de participación. (55 alumnos, equivalente a toda la rotación de Medicina Interna del Hospital Clinic de Barcelona).

El cuestionario de satisfacción fue respondido por los estudiantes al finalizar cada taller, donde se les consultó su satisfacción percibida, considerando dos grandes áreas: a) Satisfacción personal con la actividad y b) Utilidad percibida para su aprendizaje de cada una de las etapas del modelo (Figura 2).

La satisfacción percibida, medida en los estudiantes de la Universidad de Barcelona fue alta. Los alumnos calificaron positivamente, con una media de 4,98, "La atención ofrecida por los profesores de la simulación" y "Reflexionar sobre el caso en el debriefing" (Figura 3).

En relación a la evaluación psicométrica del 
A. Satisfacción personal con la actividad: Como alumno, indica tu acuerdo sobre
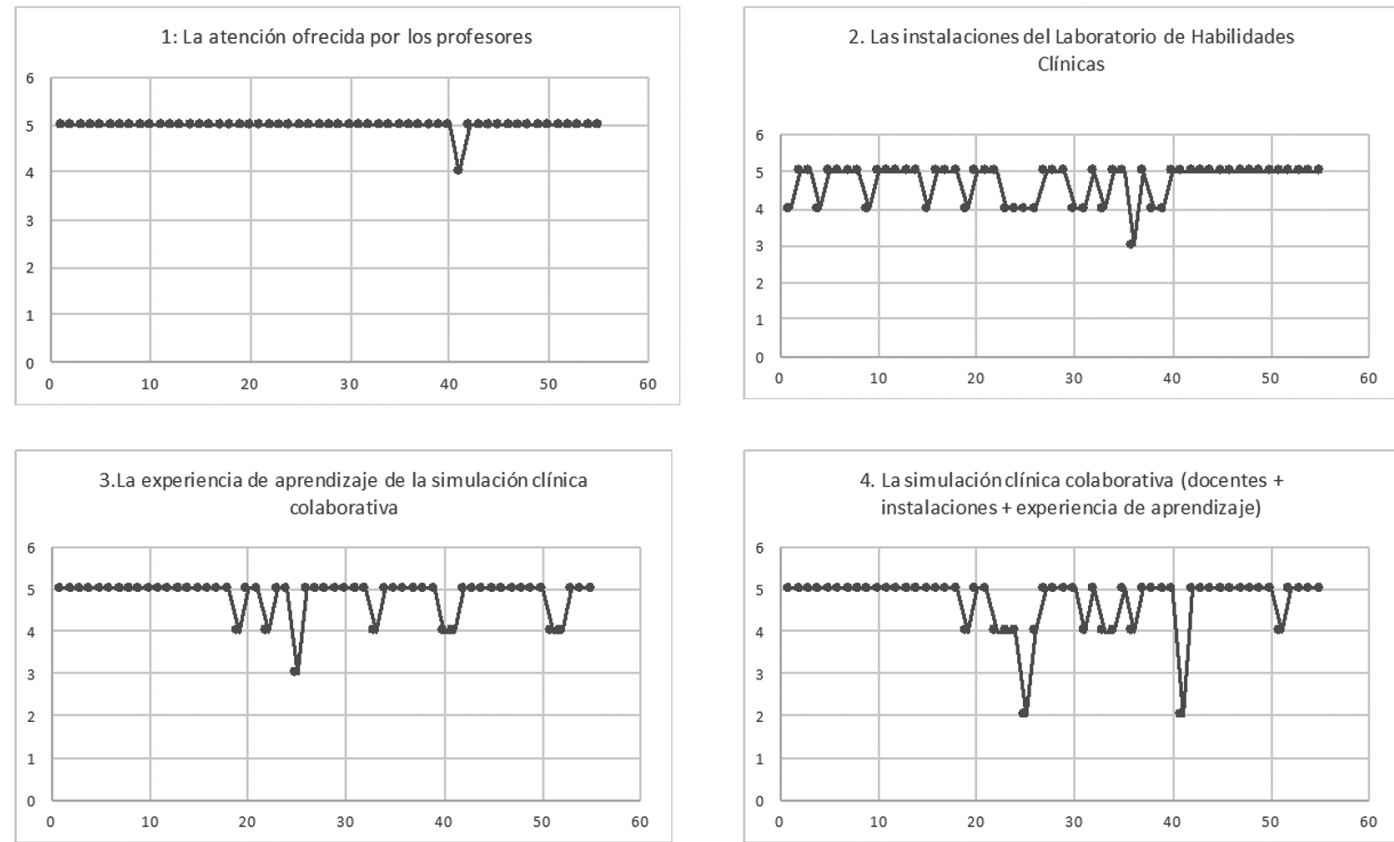

\section{B. Utilidad de las etapas para tu aprendizaje}
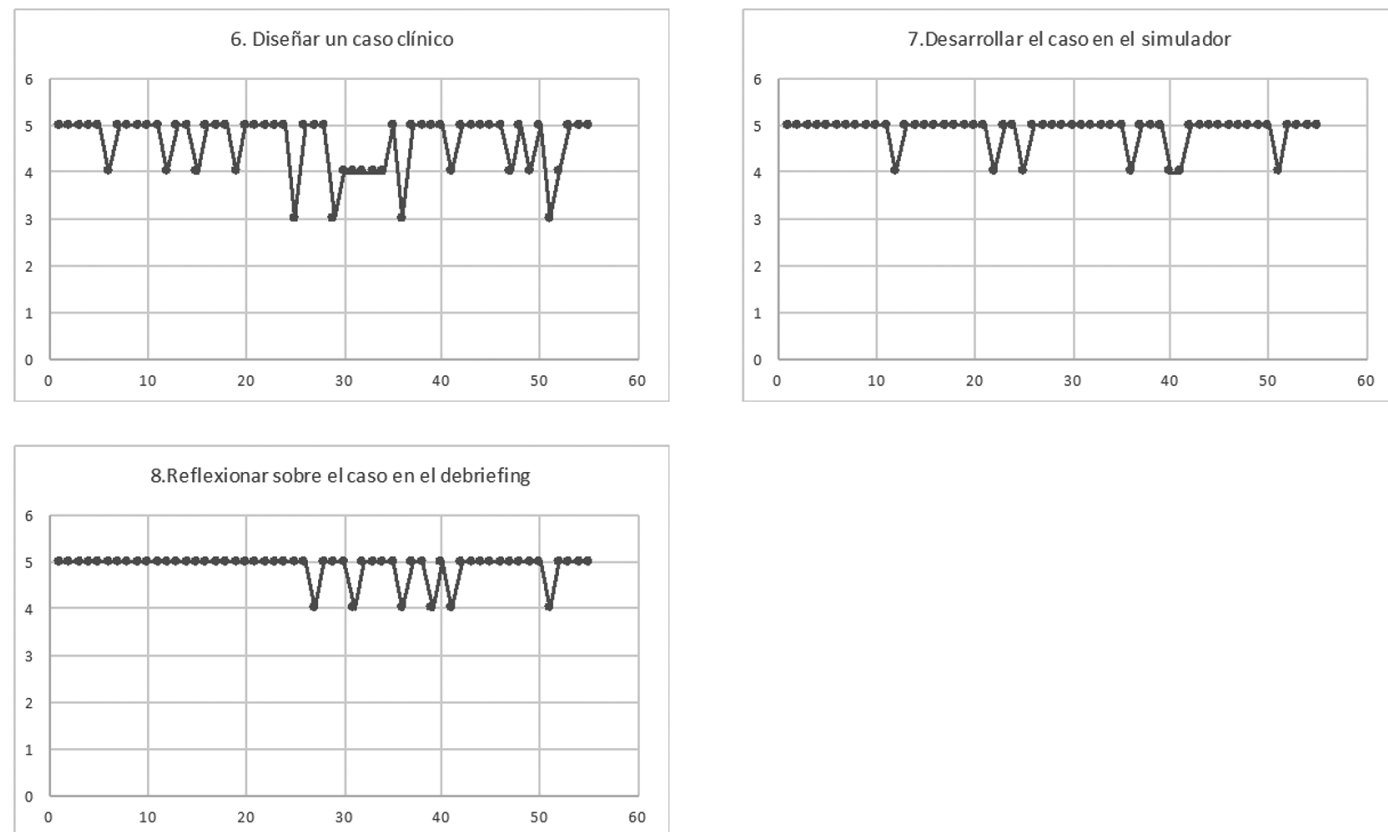

Figura 3. Satisfacción percibida por los alumnos en el taller de simulación colaborativo. 
Tabla 1. Índices psicométricos de los ítems del cuestionario de satisfacción aplicado en la Universidad de Barcelona

\begin{tabular}{|c|c|c|c|c|c|c|}
\hline Ítems Cuestionario de Satisfacción & Min & Max & Media & Desv & Varianza & $\begin{array}{l}\text { Confiabilidad } \\
\text { sin el ítem }\end{array}$ \\
\hline $\begin{array}{l}\text { 1. La atención ofrecida por los profesores de la } \\
\text { simulación }\end{array}$ & 4 & 5 & 4,98 & 0,14 & 0,02 & 0,78 \\
\hline $\begin{array}{l}\text { 2. Las instalaciones del Laboratorio de Habilidades } \\
\text { Clínicas }\end{array}$ & 3 & 5 & 4,71 & 0,50 & 0,25 & 0,78 \\
\hline $\begin{array}{l}\text { 3. La experiencia de aprendizaje de la simulación } \\
\text { clínica colaborativa }\end{array}$ & 3 & 5 & 4,84 & 0,42 & 0,18 & 0,72 \\
\hline $\begin{array}{l}\text { 4. La simulación clínica colaborativa (docentes }+ \\
\text { instalaciones }+ \text { experiencia de aprendizaje) }\end{array}$ & 2 & 5 & 4,71 & 0,66 & 0,43 & 0,68 \\
\hline 5. Diseñar un caso clínico & 3 & 5 & 4,62 & 0,62 & 0,39 & 0,73 \\
\hline 6. Desarrollar el caso en el simulador & 4 & 5 & 4,87 & 0,34 & 0,11 & 0,73 \\
\hline 7. Reflexionar sobre el caso en el debriefing & 4 & 5 & 4,89 & 0,32 & 0,10 & 0,76 \\
\hline
\end{tabular}

Tabla 2. Codificación de respuestas en la pregunta ¿Qué ventaja(s) formativa(s) de la simulación clínica colaborativa has percibido?

\begin{tabular}{|c|c|c|c|c|c|c|}
\hline Categoría & Código & Descripción & Cuenta & $\begin{array}{c}\% \\
\text { Códigos }\end{array}$ & $\begin{array}{l}\text { Participantes } \\
\text { que lo } \\
\text { mencionan }\end{array}$ & $\begin{array}{l}\% \text { de partici- } \\
\text { pantes que lo } \\
\text { mencionan }\end{array}$ \\
\hline $\begin{array}{l}\text { Plan de } \\
\text { formación }\end{array}$ & $\begin{array}{l}\text { Déficit } \\
\text { práctico }\end{array}$ & $\begin{array}{l}\text { Enfrentar escenarios prácti- } \\
\text { cos donde tenga que tomar } \\
\text { decisiones }\end{array}$ & 19 & $14,4 \%$ & 17 & $30,9 \%$ \\
\hline $\begin{array}{l}\text { Plan de } \\
\text { formación }\end{array}$ & $\begin{array}{l}\text { Integración de } \\
\text { conocimientos }\end{array}$ & $\begin{array}{l}\text { Aplicar competencias que son } \\
\text { explicadas y enseñadas de } \\
\text { forma teórica }\end{array}$ & 30 & $22,7 \%$ & 27 & $49,1 \%$ \\
\hline $\begin{array}{l}\text { Plan de } \\
\text { formación }\end{array}$ & $\begin{array}{l}\text { Incorporar } \\
\text { simulación } \\
\text { clínica }\end{array}$ & $\begin{array}{l}\text { Incorporación de talleres de } \\
\text { simulación clínica dentro de } \\
\text { las prácticas de la carrera de } \\
\text { medicina }\end{array}$ & 26 & $19,7 \%$ & 25 & $45,5 \%$ \\
\hline $\begin{array}{l}\text { Trabajo en } \\
\text { equipo }\end{array}$ & $\begin{array}{l}\text { Situación de } \\
\text { estrés }\end{array}$ & $\begin{array}{l}\text { Enfrentarse a una situación } \\
\text { de estrés y solucionarla den- } \\
\text { tro de un equipo de atención }\end{array}$ & 16 & $12,1 \%$ & 16 & $29,1 \%$ \\
\hline $\begin{array}{l}\text { Trabajo en } \\
\text { equipo }\end{array}$ & $\begin{array}{l}\text { Trabajo } \\
\text { colaborativo }\end{array}$ & $\begin{array}{l}\text { Coordinación, comunicación } \\
\text { y ser parte de un equipo de } \\
\text { trabajo }\end{array}$ & 20 & $15,2 \%$ & 19 & $34,5 \%$ \\
\hline $\begin{array}{l}\text { Trabajo en } \\
\text { equipo }\end{array}$ & $\begin{array}{l}\text { Debriefing } \\
\text { colaborativo }\end{array}$ & $\begin{array}{l}\text { Debriefing estructurado para } \\
\text { que los participantes puedan } \\
\text { reflexionar y discutir del caso } \\
\text { clínico aplicado. Discusión } \\
\text { entre pares moderada por el } \\
\text { instructor }\end{array}$ & 10 & $7,6 \%$ & 10 & $18,2 \%$ \\
\hline Aprendizaje & $\begin{array}{l}\text { Aprender del } \\
\text { error }\end{array}$ & $\begin{array}{l}\text { Aprender a equivocarse, del } \\
\text { error en una situación si- } \\
\text { mulada }\end{array}$ & 2 & $1,5 \%$ & 2 & $3,6 \%$ \\
\hline Aprendizaje & $\begin{array}{l}\text { Tomar } \\
\text { decisiones }\end{array}$ & $\begin{array}{l}\text { Tomar decisiones siendo par- } \\
\text { te de un equipo de urgencia }\end{array}$ & 6 & $4,5 \%$ & 5 & $9,1 \%$ \\
\hline Aprendizaje & $\begin{array}{l}\text { Valorar al } \\
\text { paciente }\end{array}$ & $\begin{array}{l}\text { Evaluar constantemente al } \\
\text { paciente, su estado e historia } \\
\text { clínica }\end{array}$ & 3 & $2,3 \%$ & 3 & $5,5 \%$ \\
\hline
\end{tabular}




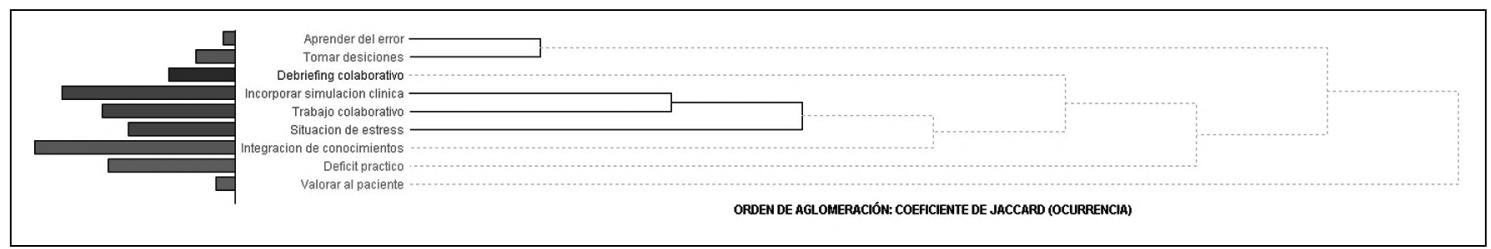

Figura 4. Orden de aglomeración: Coeficiente Jaccard (Ocurrencia), recogido del análisis de respuestas (texto libre) de 55 alumnos de la Carrera de Medicina de la Universidad de Barcelona.

cuestionario de satisfacción, la media de todos los ítems fue alta, acercándose al máximo $(5,0)$, mientras que la varianza se mantuvo baja (Tabla 1), indicando que la mayoría de los estudiantes estuvo de acuerdo con los ítems propuestos a evaluar. En general, los estudiantes manifestaron estar satisfechos con los talleres, considerando a los profesores, metodologías e infraestructura.

Considerando la validez del constructo a evaluar (satisfacción), el valor del índice KaiserMeyer-Olkin (KMO) ${ }^{31,32}$ fue 0,66, superando lo aceptable que es 0,6. El índice Bartlett, que representa el test de esfericidad ${ }^{33}$ con significancia estadística $(\mathrm{p}<0,001)$. Esto evidencia una adecuada correlación entre los ítems y una correcta adecuación muestral del estudio, respectivamente ${ }^{34}$.

El índice de confiabilidad, calculado a través del alfa de Cronbach $^{35}$ fue de 0,77; evidenciando una buena consistencia interna.

La codificación de respuestas en la pregunta ¿Qué ventaja(s) formativa(s) de la simulación clínica colaborativa has percibido?, la Tabla 2 presenta la ocurrencia de cada código en el texto de los participantes. Para su cálculo se utilizó el software de análisis de datos cualitativos QDA Miner ${ }^{36}$. En el dendograma de la Figura 4 se representa el orden de aglomeración, graficando el coeficiente Jaccard $^{37}$, que mide el grado de similitud entre dos conjuntos, sea cual sea el tipo de elementos. En este caso evaluamos los códigos del texto libre. En la figura se representan los códigos Integración de conocimientos, Incorporar simulación clínica y Trabajo colaborativo como los más mencionados por los alumnos.

\section{Discusión}

El método de simulación clínica colaborativa propuesto y aplicado posiciona a los participantes en una experiencia clínica simulada de alto rea- lismo, considerando que un médico es parte de un entorno y en múltiples oportunidades debe trabajar en equipo, con presión, tomar decisiones y resolver múltiples conflictos. Además, es necesario, a modo de discusión, listar los beneficios y restricciones que entregaría la unión de ambos paradigmas de enseñanza-aprendizaje.

Las fortalezas son esencialmente tres.

1. Los alumnos diseñan un caso clínico, son instructores y posteriormente son médicos tratantes de otro caso. Esto hace que el estudiante deba redoblar esfuerzos: 1) Para estudiar, conocer y diseñar bien el caso del que serán instructores y 2) Para tratar y desenvolverse adecuadamente como equipo médico tratante el caso diseñado por sus compañeros.

2. Eficiencia en consumo de tiempo. La relación tiempo/número de alumnos, permite en $3 \mathrm{~h}$, con 1 profesor, enseñar a un grupo de 15 alumnos 3 diagnósticos diferentes (en forma activa y participativa). Además, debemos considerar la alta escalabilidad y facilidad de aplicación. Si se consideran 2 profesores, en 4 h podría incluir el doble (30 alumnos) sin mermar la calidad del método.

3. Eficiencia de recursos: Es aplicable con cualquiera de los recursos disponibles de simulación: con y sin un simulador robotizado, con actores estandarizados o con los mismos participantes (grupo diseñador) que actúen de paciente.

Las limitaciones son, principalmente, dos y deben ser superadas para validar el método:

1. Precisa la evaluación de la eficacia: Se debe evaluar su efecto sobre el aprendizaje, considerando cohortes de aplicación del método y evaluando su rendimiento posteriormente en un Examen Clínico Objetivo y Estructurado $(\mathrm{ECOE})^{38,39}$. 
2. Precisa instrumentos estandarizados: La aplicación requiere el diseño y validación de un conjunto de instrumentos estandarizados que permitan medir la satisfacción y las interacciones del grupo, esto último debe ser investigado con mayor profundidad.

La aplicación del modelo en Chile y España abre un amplio horizonte en la forma de ver las actividades prácticas en alumnos de medicina. El nivel de satisfacción fue alto y las opiniones recogidas reflejan la motivación y la necesidad de seguir con este tipo de metodologías. Durante el primer semestre del año 2018, seguirá su aplicación en ambas escuelas de Medicina con el objetivo comparar resultados y seguir perfeccionando el modelo.

\section{Referencias}

1. Paice E, Heard S. Collaborative learning. Med Educ 2003; 37 (9): 758-9.

2. Lerner S, Magrane D, Firedman E. Teaching Teamwork in Medical Education. Mt Sinai J Med A J Transl Pers Med 2009; 76 (4): 318-29.

3. Bleetman A, Dale T, Brace SJ. Human factors and error prevention in emergency medicine. Emerg Med J 2012; 29 (5): 389-93.

4. Kohn LT, Corrigan JM, Donaldson MS. To Err Is Human:: Building a Safer Health System (Vol. 6). Washington, DC: National Academies Press; 2000.

5. Makary M, Daniel M. Medical error-the third leading cause of death in the US. BMJ 2016; 353: i2139.

6. Khan K, Pattison T, Sherwood M. Simulation in medical education. Med Teach $2011 ; 33$ (s1): 1-3.

7. Okuda Y, Bryson EO, DeMaria S, Jacobson L, Quiñones J, Shen B, et al. The utility of simulation in medical education: what is the evidence? Mt Sinai J Med A J Transl Pers Med 2009; 76 (4): 330-43.

8. Palés-Argullós J, Gomar-Sancho C. El uso de las simulaciones en educación médica. Educ Knowl Soc 2010; 11 (2): 147-70.

9. Gaba DM. The Future Vision of Simulation in Healthcare. Simul Healthc 2013; 35: e1511-30.

10. McGaghie WC, Issenberg SB, Petrusa ER, Scalese RJ. A critical review of simulation-based medical education research: 2003-2009. Med Educ 2010; 44 (1): 50-63.

11. Motola I, Devine LA, Chung HS, Sullivan JE, Issenberg SB. Simulation in healthcare education: a best evidence practical guide. AMEE Guide No. 82. Med Teach 2013; 35 (10): e1511-30.
12. Issenberg SB. The scope of simulation-based healthcare education. Simul Healthc 2006; 1 (4): 203-8.

13. Guinez-Molinos S, Molina AM, Sancho CG, Arias V, Szyld D, García Garrido E, et al. A collaborative clinical simulation model for the development of competencies by medical students. Med Teach 2016; 1-8.

14. Prion S. A Practical Framework for Evaluating the Impact of Clinical Simulation Experiences in Prelicensure Nursing Education. Clin Simul Nurs 2008; 4 (3): 69-78.

15. Bremner MN, Aduddell K, Bennett DN, Vangeest JB. The Use of Human Nursing Students. Nurse Educ 2006; 31 (4): 170-4.

16. Levett-Jones T, Mccoy M, Lapkin S, Noble D, Hoffman $\mathrm{K}$, Dempsey J, et al. Nurse Education Today The development and psychometric testing of the Satisfaction with Simulation Experience Scale. Ynedt 2011; 31 (7): 705-10.

17. Weller JM, Nestel D, Marshall SD, Brooks PM, Conn JJ. Simulation in clinical teaching and learning. Med J Aust 2012; 196 (9): 594.

18. Edmunds S, Brown G. Effective small group learning: AMEE Guide No. 48. Med Teach 2010; 32 (9): 715-26.

19. Crosby J. Learning in small groups. Med Teach 2013; 18 (3): 189-202.

20. Stahl G, Koschmann T, Suthers D. Computer-supported collaborative learning: An historical perspective. In: Cambridge handbook of the learning sciences. 2006. p. 409-26.

21. Wasson B. Computer Supported Collaborative Learning: an overview. Lect notes from IVP. 1998; 482.

22. Lipponen L. Exploring Foundations for Computer-Supported Collaborative Learning. Proc Conf Comput Support Collab Learn Found a CSCL community, Int Soc Learn Sci 2002; 72-81.

23. Dillenbourg P, Järvelä $S$, Fischer F. The evolution of research on computer-supported collaborative learning. In: In Technology-enhanced learning. 2009. p. 3-19.

24. Keynejad RC. Global health partnership for student peer-to-peer psychiatry e-learning: Lessons learned. Global Health 2016; 12 (1): 82.

25. Steinert Y, Naismith L, Mann K. Faculty development initiatives designed to promote leadership in medical education. A BEME systematic review: BEME Guide No. 19. Med Teach 2012; 34 (6): 483-503.

26. Dillenbourg P, Baker MJ, Blaye A, O’Malley C. The evolution of research on collaborative learning. Technol Learn 1995; 3-19.

27. Dillenbourg P. What do you mean by collaborative learning? Collab Cogn Comput approaches 1999; 1: 1-15.

28. Stahl G, Koschmann T, Suthers D. Computer- supported collaborative learning: An historical perspective 2014; (2006): 1-17. 
29. Maestre JM, Sancho R, Rábago JL, Martínez A, Rojo E, Moral ID. Diseño y desarrollo de escenarios de simulación clínica: análisis de cursos para el entrenamiento de anestesiólogos. FEM Fund Educ Médica 2013; 16 (1): 49-57.

30. Center for Medical Simulation. Debriefing by CMS [Internet]. 2016. Available from: https://harvardmedsim. org/resources-other.php

31. Kaiser HF. An index of factorial simplicity. Psychometrika 1974; 39 (1): 31-6.

32. Kaiser HF. A second generation little jiffy. Psychometrika 1970; 35 (4): 401-15.

33. Bartlett M. A note on the multiplying factors for various $\chi^{2}$ approximations. J R Stat Soc Ser B 1954; 269-98.

34. Rotthoff T, Ostapczuk M, Bruin J De. Assessing the lear- ning environment of a faculty: psychometric validation of the German version of the Dundee Ready Education Environment Measure with students. Med Teach 2011; 33 (11): e624-36.

35. Cronbach L. Essentials of psychological testing. 1949.

36. Research-Provalis. Software QDA Miner [Internet]. 2016. Available from: https://provalisresearch.com/es/ products/software-de-analisis-cualitativo/

37. Real R, Vargas J. The probabilistic basis of Jaccard's index of similarity. Syst Biol 1996; 45 (3): 380-5.

38. Harden RM, Stevenson M, Downie WW, Wilson GM. Assessment of clinical competence using objective structured examination. Br Med J 1975; 1 (5955): 447-51.

39. Harder B. Use of simulation in teaching and learning in health sciences: a systematic review. J Nurs Educ 2009. 